\title{
Magnetic Resonance Imaging of Gliomas
}

\author{
Pilar López-Larrubia, Eva Cañadillas-Cárdenas, \\ Ana M. Metelo, Nuria Arias, Miguel Martínez-Maestro, \\ Aire Salguero and Sebastián Cerdán \\ Instituto de Investigaciones Biomédicas "Alberto Sols", CSIC-UAM \\ Spain
}

\section{Introduction}

Brain cancer is a life threatening neurological disorder in which malignant cells, grow, proliferate and invade the original cerebral structures of the host, hampering seriously adequate brain function. Malignant cells generate eventually a dedifferentiated tumoral mass that interferes with vital brain functions as sensory and motor activations, memory and perception and neuroendocrine regulation, among others. The fully developed tumoral mass consumes a significant part of cerebral volume resulting in cerebral compression and serious neurological impairments, such as vision or hearing disturbances and eventually lethal cerebrovascular complications. Most brain tumors remain asymptomatic during early development, revealing their symptoms and lethal nature only at later stages. Therapy is facilitated many times by an early finding, a circumstance making the neuroimaging approaches particularly useful in the detection and handling of these lesions.

In the last decades, Magnetic Resonance Imaging (MRI) approaches have evolved into the most powerful and versatile imaging tool for brain tumor diagnosis, prognosis, therapy evaluation, monitoring of disease progression and planning of neurosurgical strategies. MRI methods enable the non invasive assessment of glioma morphology and functionality providing a point of likeness into histopathological grading of the tumor and helping in this way a more successful patient management. This impressive evolution is based not only for the high resolution and quality of the anatomical images obtained, but on the additional possibilities to achieve quantitative functional information on tumoral physiopathology and its repercussions in the sensorial, motor and integrative functions through the brain. The use of conventional paramagnetic or superparamagnetic contrast media allows for the identification of areas with blood-brain barrier (BBB) disruption and the recent molecular imaging approaches enable researchers to visualize molecular events associated to tumor proliferation and invasion, bringing the potentials of diagnostic imaging to the cellular and molecular aspects of tumor biology. Moreover, functional MRI approaches as performed in the clinic are endowed with the potential to detect and characterize the earliest neoangiogenic, metabolic and hemodynamic alterations induced by the neoplasm.

Several advanced magnetic resonance (MR) methodologies have been proposed in the last years to assess the functional competence in healthy and pathologic brain tissue. Diffusion and perfusion MRI are probably the two main approaches that have reached a relevant clinical role 
in brain oncology, particularly in neovascular imaging (Calli, Kitis et al. 2006). The diffusion approaches investigate the thermally induced random molecular motions of water molecules in tissues. The perfusion phenomenon describes and quantifies the microvascular blood flow which feeds a volume element of an organ or tissue. At a first glance these two phenomena seem to be very different, however a closer look identifies they both concerned with underlying molecular motions. Moreover, the random distribution of capillaries in tissues such as brain, provides tissue perfusion with some of the characteristic features of diffusive motion.

This chapter will summarize the arsenal of MRI approaches providing functional information on gliomas based on diffusion and perfusion MRI methods. We begin with a description of the pathological classification of gliomas and its implications for MRI diagnosis, continuing with the applications of diffusion weighted imaging (DWI), diffusion tensor imaging (DTI) and tractography to the characterization of these brain tumors. We conclude showing the MRI methodologies available for the evaluation of cerebral and tumoral perfusion, addressing the use of Dynamic Susceptibility Contrast (DSC), Dynamic Contrast Enhanced (DCE) MR images and Arterial Spin Labelling (ASL). Our review focuses mainly in the clinical applications of these methodologies, providing a brief introduction into the physical principles of each one. For interested readers, the following review articles provide more detailed descriptions of the corresponding physical principles of diffusion weighted (DW) MRI (Mori and Barker 1999), DTI (Basser and Jones 2002) and Tractography (Mori and van Zijl 2002) or the different approaches to investigate microvascular perfusion (Ostergaard 2005; Detre, Wang et al. 2009; Larsson, Courivaud et al. 2009).

\section{Histological classification of gliomas. Implications in MRI diagnosis}

Glioma is a non-specific term, broadly suggesting that the observed tumor originates from glial cells. These tumors account for more than $85 \%$ of primary brain neoplasms classified histologically, immunohistochemically and structurally as astrocytomas, ologodendrogliomas or oligoastrocytomas, depending on their morphological, immunochemical or molecular features. Gliomas are assigned a grade depending on their histolopathological properties (figure 1). The grade provides insight into the growth rate of the tumor. According to the classification of the World Health Organization (WHO), tumors in grade I depict the lowest malignancy, their cells look nearly normal and the tumor grows slowly. Grade II tumors are also slow growing, but show a slightly abnormal microscopic appearance. Grade III tumors are malignant by definition. Their cells look very different from the original neural cells and are actively proliferating. Grade IV is the most malignant and aggressive, defined by galloping genetic instability, complete morphological and metabolic reprogramming, fast and uncontrolled proliferation, intense resistance to apoptosis, diffuse infiltration, robust angiogenesis and propensity to necrosis. From this group Glioblastoma multiforme (GBM) is the most frequent, aggressive and lethal intracranial tumor, reaching approximately $50 \%$ of all astrocytomas (Furnari, Fenton et al. 2007). Despite decades of intensive research, high-grade gliomas (WHO grade III and IV) are currently estimated incurable with a poor or very poor survival, up to one year in glioblastoma and from two to three years in anaplastic astrocytomas (Reardon, Rich et al. 2006).

The tumor grade determines the most appropriate chemotherapeutic or surgical treatment, but histology, requiring a tumor biopsy, remains currently the only method able to yield 
unambiguously this information. In spite of this, many of the histopathological features of gliomas are amenable to MRI explorations. In particular, the growth rate and the appearance of necrotic zones may be followed by sequential morphological MRI examinations, the cellularity and tumor microstucture investigated by diffusion weighted MRI, the compression effects of the tumor on surrounding neuronal tracts of the white matter may be examined by MRI tractography from DTI approaches and the development of the neoangiogenic vasculature characterized by perfusion imaging. These properties entail the MRI method with unprecedented capabilities to asses non invasively glioma grade and its potential repercussions. In the following sections we shall provide a description of main physical principles underlying these methodologies and provide illustrative examples on their applications.

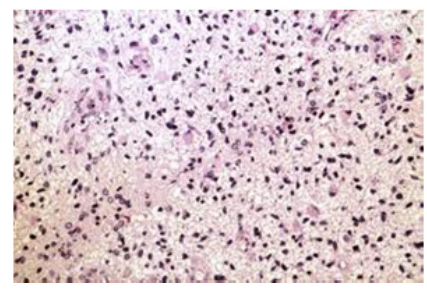

(a)

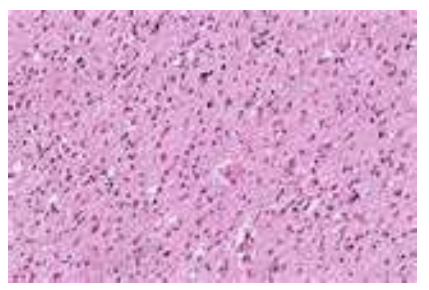

(b)

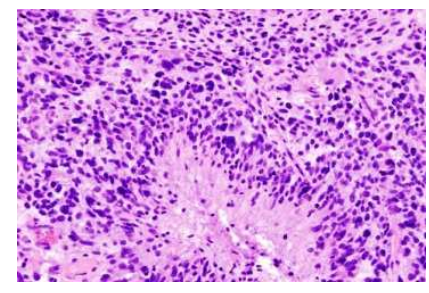

(c)

Fig. 1. Histopathological features from gliomas of increasing grade as revealed by haematoxylin/eosin staning. (a) WHO grade II astrocitoma , (b) WHO grade III anaplastic astrocytoma and (c) WHO grade IV glioblastoma multiforme

\section{Diffusion magnetic resonance imaging}

The diffusion phenomenon describes quantitatively the random (Brownian) molecular motion translations of water molecules originating ultimately from thermal energy. In a tissue, like the brain, the mechanism of diffusion involves mainly the motion of water molecules on a scale of 5 to 15 micrometers over the approximate $40 \mathrm{~ms}$ of measurement time. It is described by an Apparent Diffusion Coefficient (ADC), representing the average translational displacement of a water molecule during a time unit. The diffusion process has a vectorial nature, involving magnitude and direction. Its magnitude and direction in vivo depend on a variety of factors including permeability barriers and their spacing, microviscosity of the intracellular milieu, molecular obstructions to water displacements, duration of the diffusion measurement and, eventually the bulk flow within capillaries and water active transporters in tissue. These circumstances make the interpretation of diffusion measurements complex, but at the same time highly informative on all the aspects influencing it.

In vivo Diffusion Weighted Imaging (DWI) provides images with an inherent contrast different from that obtained by conventional structural MR techniques. In this sense, DWI gives unique information about the microstructure and viability of brain tissue, with the signal intensity of the image depending on the average translational motion of water molecules, an aspect known to be appreciably modified in cerebral tumors (Schaefer, Grant et al. 2000).

\subsection{Apparent Diffusion Coefficient}

The first description of a diffusion study was provided by Stejskal and Tanner, who used a spin-echo acquisition protocol in which two gradient pulses equal in magnitude but 
opposite in directions (figure 2) were intercalated before and after the 180 degrees refocusing pulse, thus allowing the measurement of molecular water motions in the direction of the applied gradient (Stejskal and Tanner 1965).

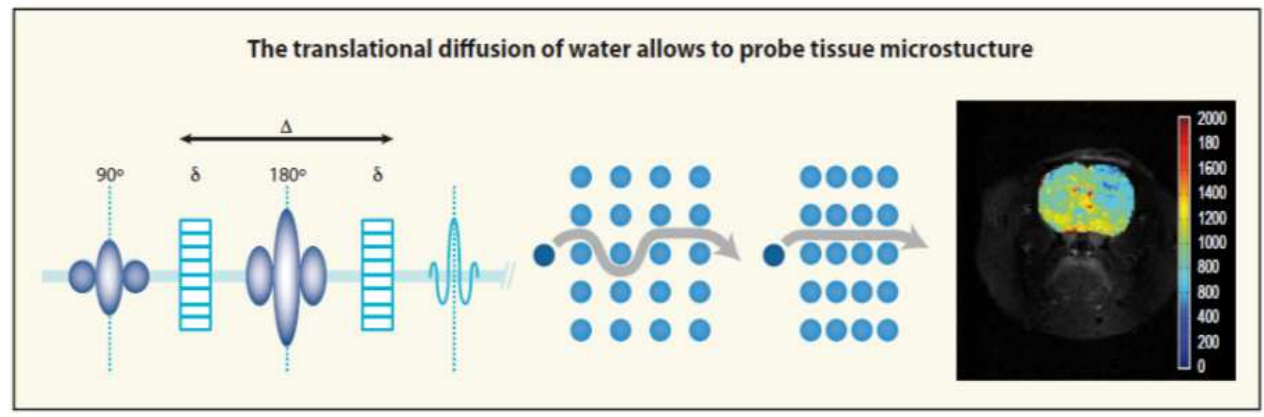

Fig. 2. Diffusion Weighted MR Imaging. Left: The Stejskal-Tanner pulse sequence. The basic spin-echo sequence is sensitized to diffusion using two phase gradients of duration $\delta$, separated by the diffusional time $\Delta$, located symmetrically before and after the 180 pulse. Center: Influence of cellularity on the translational diffusion measurements. Note that in the same time, a water molecule will move translationally a shorter path in an increased cellular density and molecularly more crowded medium. A representative image of the ADC measured in the normal rat brain (Pacheco-Torres, Calle et al.). Reproduced with permission

Because of the same magnitude and the opposite directions of these two gradients, the MR signal of water molecules without net motion will be identical to its intensity as obtained without diffusion gradients in the same cerebral location. However, for locations showing net translational motion of water molecules in the direction of the applied gradient, the opposing effects of the two gradients will not be cancelled, and the resulting image will depict lower intensity in these regions. Signal intensity will be smaller, the faster the average movement of water molecules or the larger ADC. This leads to a DW image depicting hypointensity in the tissue voxels containing faster moving water molecules and hiperintensity in those with slower water displacements.

Under these conditions, the MR signal loss is related to: (i) the diffusion coefficient of the water and (ii) the duration and strength of the magnetic field gradients used in the measurement. The following equation applies:

$$
\mathrm{SI}=\mathrm{SI}_{0} \times \exp (-b \times \mathrm{ADC})
$$

where; $\mathrm{SI}_{0}$ represents the signal intensity for each pixel in the absence of gradient pulses, $b$ is an experimentally modifiable parameter including the duration $\delta$, intensity and delay separation $\Delta$ of the applied magnetic field gradients and ADC is the apparent diffusion coefficient of the water in the investigated tissue voxel. Using images acquired with increasing $b$ values and thus, increasingly sensitized to diffusion, a parametric map can be calculated by plotting for every pixel the linear relationship between the natural logarithm of the relative signal loss $\left(\mathrm{SI}_{/} \mathrm{SI}_{0}\right)$ versus $b$. This affords the calculation of the corresponding ADC on a pixel-by-pixel base, representing then every pixel value in the resulting ADC map. 
In vivo measurements of ADC in the human brain by using MRI are well documented both in physiological and physiopathological conditions (Le Bihan, Breton et al. 1986; Thomsen, Henriksen et al. 1987; Chien, Buxton et al. 1990; Hajnal, Doran et al. 1991; Schaefer, Grant et al. 2000). Regions of increased or decreased diffusion due to pathology can be clearly identified in a diffusion weighted MRI study by the corresponding changes in intensity, either in the diffusion weighted images or in fitted ADC maps.

\subsection{Diffusion weighted imaging of gliomas}

Although DWI studies were initially focused on stroke (Warach, Chien et al. 1992), multiple sclerosis (Larsson, Thomsen et al. 1992) and hydrocephalus (Gideon, Thomsen et al. 1994), an increasing number of diffusion studies on brain is being performed.

In 1994 Tien et al. acquired diffusion images in patients with high-grade gliomas using Echo Planar Imaging (EPI) techniques to measure ADC along cephalocaudal axis, comparing the obtained results with those found by using conventional (SE) or fast spin-echo (FSE) MRI (Tien, Felsberg et al. 1994). The ADC images enabled these authors to differentiate various regions within the tumor, concluding that echo-plannar DWI appeared a more powerful tool in the characterization of brain neoplasm than conventional techniques.

In 1995 Brunberg et al. reported their study performed with a motion-insensitive spin-echo sequence measuring ADC along three orthogonal axes (Brunberg, Chenevert et al. 1995). They aimed to determine if there was a relationship between water diffusion coefficients or its anisotropy in MR-defined regions of normal and abnormal parenchyma in patients with cerebral gliomas. They were able to distinguish between normal white matter, cystic or necrotic areas, regions of edema and solid enhancing tumor by comparing anisotropic ADC values in those regions.

In 1997 Krabbe et al. measured ADC in different regions of patients with brain tumors (Krabbe, Gideon et al. 1997). They reported diffusion values in contrast-enhancing tumor, non-enhancing tumor, cyst or necrosis, edema and cerebro-spinal fluid (CSF) for malignant gliomas, low-grade gliomas, metastases, meningiomas and cerebral abscess. They found that mean ADC in contrast-enhancing tumor was significantly higher in metastases than in high-grade gliomas, while no significant differences were found between ADC in contrastenhancing tissue in meningiomas and metastases or high-grade gliomas. The highest ADC was always found in CSF. ADC in edema was higher than in contrast-enhancing tumor, and in edema around cerebral metastases was significantly higher than those around high-grade gliomas. These findings could help to distinguish on this basis high-grade gliomas and metastases prior to the surgery.

After these initial reports, a large number of results have been published demonstrating the vast possibilities of diffusion MRI in brain oncology. These cover the applications of DWI from glioma characterization and evaluation to the identification of patients with poor treatment response or tumor recurrence (Provenzale, Mukundan et al. 2006; Hamstra, Rehemtulla et al. 2007; Hamstra, Galban et al. 2008). In addition, DWI imaging may allow the non invasive grading of tumor cellularity since cells constitute barriers that restrict microscopically the motion of the water molecules within the tissue. In this sense, the diffusion of water molecules across the tumor as compared to the diffusion in normal brain tissue is expected be different, depicting different diffusivity and ADC depending on the tumor cellularity and grade. As cells constitute a barrier to water motion, tumors with higher cellularity are expected to show lower ADC values than less cellularly crowded 
tumors (Sugahara, Korogi et al. 1999; Gauvain, McKinstry et al. 2001). On the other hand, an increase in ADCs usually is correlated with decreased cellularity, revealing successful therapy and/or induced necrosis (Lyng, Haraldseth et al. 2000). Although some authors have reported lower diffusivity of water in high-grade than in low grade gliomas (Bulakbasi, Kocaoglu et al. 2003), it should be mentioned that there is a considerable overlap between ADCs in both types of neoplasm (Kono, Inoue et al. 2001). Even so, there is clearly an inverse correlation between tumor cellularity and ADC values measured in brain tumors allowing to distinguish these from other intracranial lesions. Figure 3 illustrates the important differences found in ADC of intratumoral and extratumoral regions, in two different neoplasm types. Images (c) depict ADC maps in two patients with different brain tumor types. Upper panel shows a high average ADC in the patient with partially resected GBM, while in lower pannel reveals a low average ADC in a patient with a primary CNS lymphoma. Regions of white matter edema distal to enhancing tumor margins usually show diffusion coefficient values significantly different from normal white and grey matter, and from areas of tumor cyst or necrosis, respectively. Notably, there are not appreciable differences between these ADCs from edema regions and those from enhancing tumor.

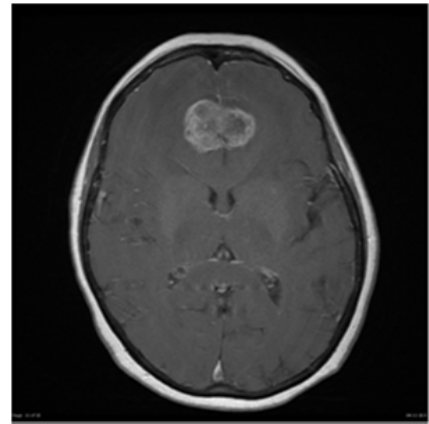

(a)

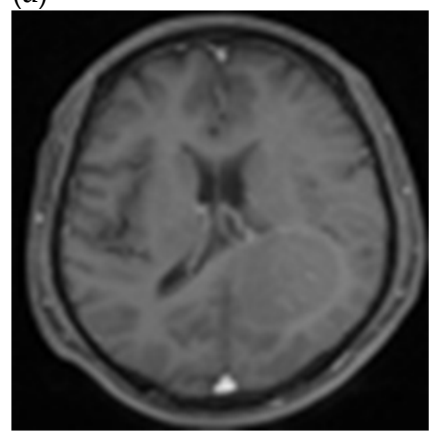

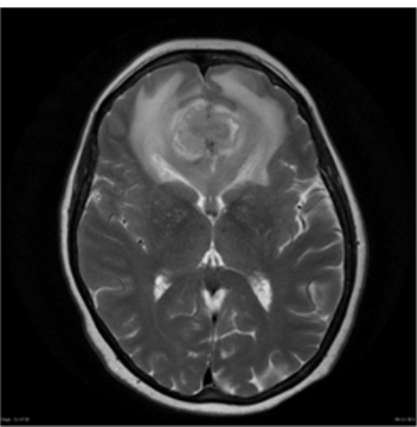

(b)

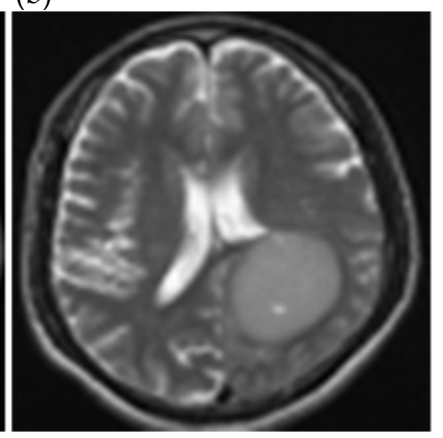

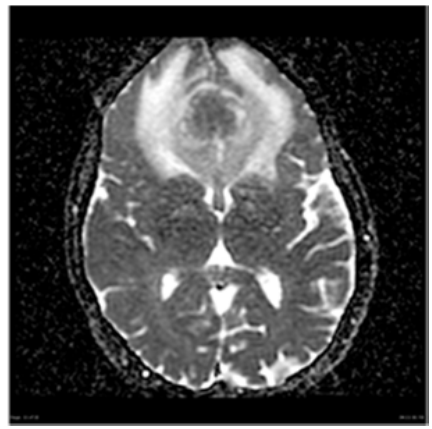

(c)

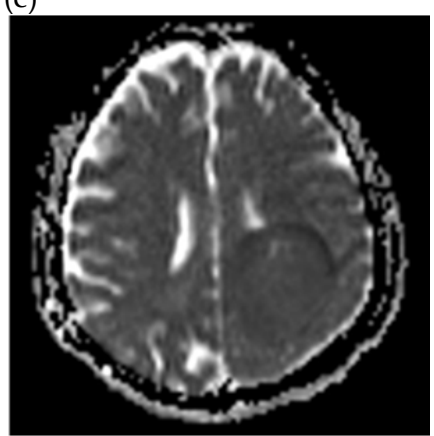

Fig. 3. Upper panels: A patient with GBM and high ADC values. (a) Axial contrast-enhanced T1W image shows a mass; (b) T2W image shows lesion and edema; (c) ADC map shows that mean ADC in tumor is much higher than in normal brain. Lower panels: A patient with primary CNS lymphoma and low ADC values. (a) Axial contrast-enhanced T1W image shows non enhancing lesion; (b) T2W image shows signal intensity in tumor darker than gray matter; (c) ADC map shows mean ADC in tumor is lower than in normal tissue. (http://radiopaedia.org/encyclopaedia/cases) Reproduced with permission. 
This is probably related with the fact that there is an increase in intracellular water in edematous white matter as well as within the tumor. Although this is true for almost all gliomas, its important to consider here, that interpretation of diffusion images and ADC values for diagnostic and prognostic purposes may be tumor type and individualdependent.

\subsection{Diffusion Tensor Imaging}

Water molecules are not able to diffuse with equal freedom in all directions through the brain, as surrounding cellular membranes and tissue structures limit to different extents their translational mobility in different directions. As a result, the ADC values measured in different directions are not equal and cerebral diffusion becomes anisotropic. This occurs mainly in white matter since molecular diffusion is relatively unrestricted along the trajectory of the neuronal axons and nerve fibres, but significantly more restricted in the perpendicular plane. The ADC in the grey matter, containing mainly neuronal bodies is more isotropic, with similar restrictions in all directions (Hajnal, Doran et al. 1991). This anisotropic behaviour of the molecular motions of water molecules in the brain can be observed by MRI obtaining images with the diffusion gradients applied in different spatial directions. The dominant direction and magnitude of water diffusion in every pixel is then obtained by calculating the resulting tensor from the ADC vectors measured in all investigated directions. This methodology is known as Diffusion Tensor Imaging (DTI), a method requiring the sampling of the ADC in six or more directions (Nucifora, Verma et al. 2007). This relatively complex mathematical modelling approach allows the preparation of three-dimensional ADC anisotropy maps, a very potent tool in the examination of the brain microstructure, inaccessible to other neuroimaging modalities. Using DTI sequences, it is possible to explore the integrity of white matter structures and detect abnormalities not visible in the conventional MRI acquisitions. DTI also allows to calculate tractography maps, revealing white matter fiber tracts, very useful to determine the infiltration or degeneration of the original fiber tracts by invading tumors (Mori and van Zijl 2002; Witwer, Moftakhar et al. 2002).

\subsubsection{Fractional Anisotropy and Mean Diffusivity}

Diffusion properties assessed by DTI, such as the fractional anisotropy (FA) and average of diffusion coefficient or mean diffusivity (MD), have become nowadays the most frequently used parametric images in neuro-oncology. Such images are correlated with cellular physiology and tissue microstructure, being extensively used to delineate glioma grades. MD measurements allow differentiation of necrotic areas within a high-grade gliomas and peritumoral edema from adjacent white matter; but they are not able to assess tumor infiltration adequately (Tropine, Vucurevic et al. 2004). On the other hand, FA values can be associated to functional tumoral characteristics, including WHO grade. So, combination of DTI related parameters in a multiparametric approach may facilitate glioma characterization and classification. In this respect, several reports aimed, at the precise elucidation of individual anisotropy patterns of brain water diffusivity which can reflect histopathological features used to graded gliomas (Jakab, Molnar et al.). Jakab et al. reported that these parameters can be used as indicators of glioma classification types and tumor physiopathology features like proliferation, metabolism or blood flow. These authors 
proved a consistent relationship between DTI-related information and histopathological data obtained from biopsies.

FA and MD are very sensitive indicators of white matter integrity also. Some clear examples of color-based images obtained from DTI acquisition are shown in figure 4 and 5, where not only edema region can be clearly distinguished from solid tumor by MD and FA color-based maps (figure 4), but also anisotropic directional maps depict those regions of fiber tract degeneration.

The diffusion MRI study of a brain tumor patient may show a lack of a significant difference in mean diffusion anisotropy between cerebral cortex and solid tumor reflecting the disruption of myelinated fiber orientation by the pathology. In cystic or necrotic regions there is not anisotropy in diffusion. However, the mass effect of a tumor can induce a displacement or distortion of fiber tracts, by mechanical compression, yielding a decrease in mean ADC values and an increase in diffusion anisotropy relative to white matter in health hemisphere as it is shown in figure 5. Moreover, a significant difference in diffusion anisotropy appears between regions of white matter edema and tumor, enhancing or not. This difference is related to the existence of intact myelin membranes within the edema region and the loss of that integrity in areas of active tumor cell proliferation. This promises useful applications in diagnosis and preoperative planning since ADC values alone do not allow the distinction between enhancing central tumor and surrounding tissue.
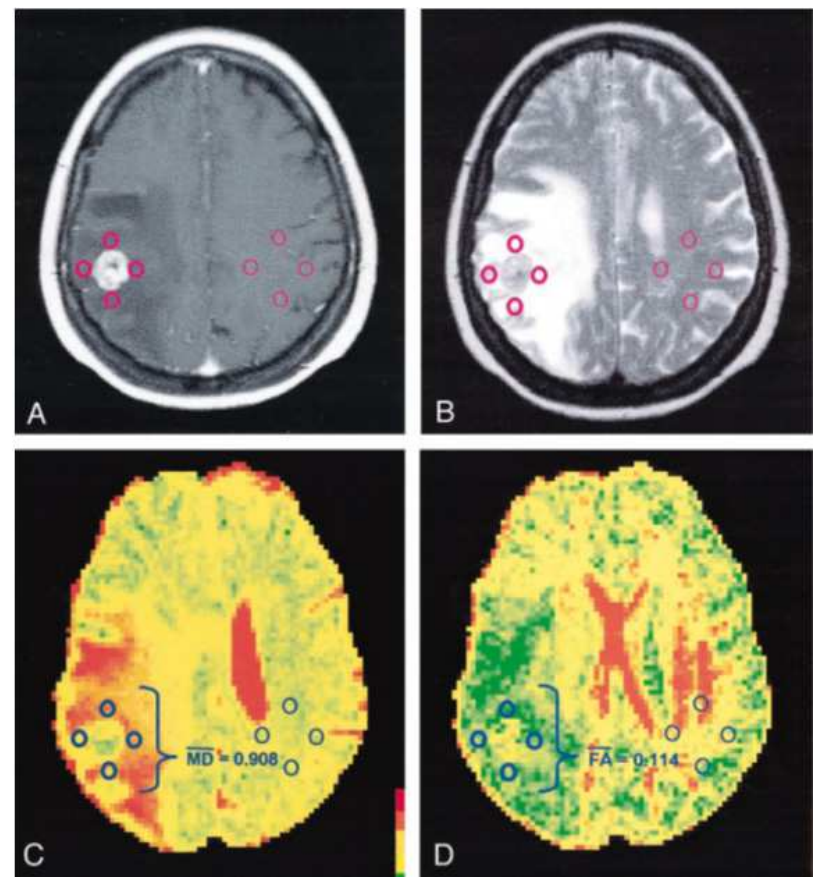

Fig. 4. Metastatic brain tumor. A: T1W image; B: T2W image; C and D show MD and FA overlay maps respectively, and mean values from peritumoral edema. ( $\mathrm{Lu}$, Ahn et al. 2003; Kinoshita, Yamada et al. 2005). Reproduced with permission 


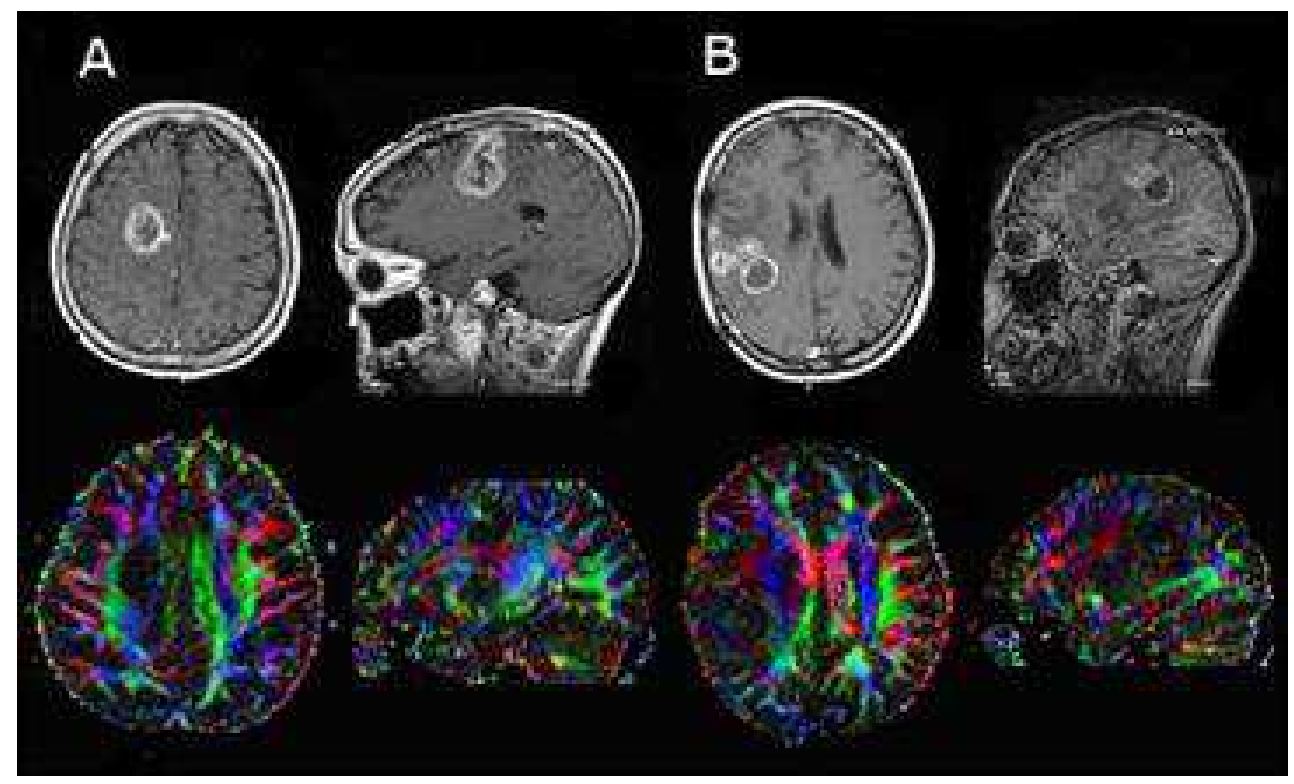

Fig. 5. T1W (upper) and relative anisotropy maps (lower). Each color indicates the principal eigenvector. Color intensity correlates with anisotropy grade. A: right frontal brain tumor; B: anaplastic ependymoma. In lower panels is clearly detected the loss of white matter integrity in both cases. (Lu, Ahn et al. 2003; Kinoshita, Yamada et al. 2005). Reproduced with permission

\subsubsection{Tractography}

Gliomas are infiltrating tumors in which the tumor cells may invade cerebral structures following the path of normal fiber tracts, making it difficult to differentiate tumoral from healthy tissue and detect the extension of white matter infiltration. Although several approaches from DTI have been reported to make the detection and reliable quantification of this diffuse tumor infiltration (Price, Burnet et al. 2003; Tropine, Vucurevic et al. 2004), this aspect still represents a very challenging question. One of the best developments to improve this situation is based in the visualization of diffusion tensor field by ellipsoids and colour coding of the main diffusion orientations (MDO) that allows the assessment of fibre bundles infiltration more reliably (Schluter, Stieltjes et al. 2005). The color maps and diffusion ellipsoids permit the identification of the particular fibre tracts bundles leading to the periphery of glioma by a reduced FA. Obviously, this approach is helping to improve therapeutic decisions and to minimize interventional risk. Additionally, it has been found that tumor recurrence has the tendency to occur along directions where the region of abnormal isotropic diffusion spreads beyond the region of abnormal anisotropic diffusion. On these grounds, it is eventually possible to predict regions of potential tumor infiltration and/or recidive by detecting abnormalities with high spatial resolution by using DTI. An aspect of increasing interest is the development of new applications that allow obtaining not only presurgical but also postsurgical information related to the tumor affected tissue for a more accurate postoperative prognosis. 

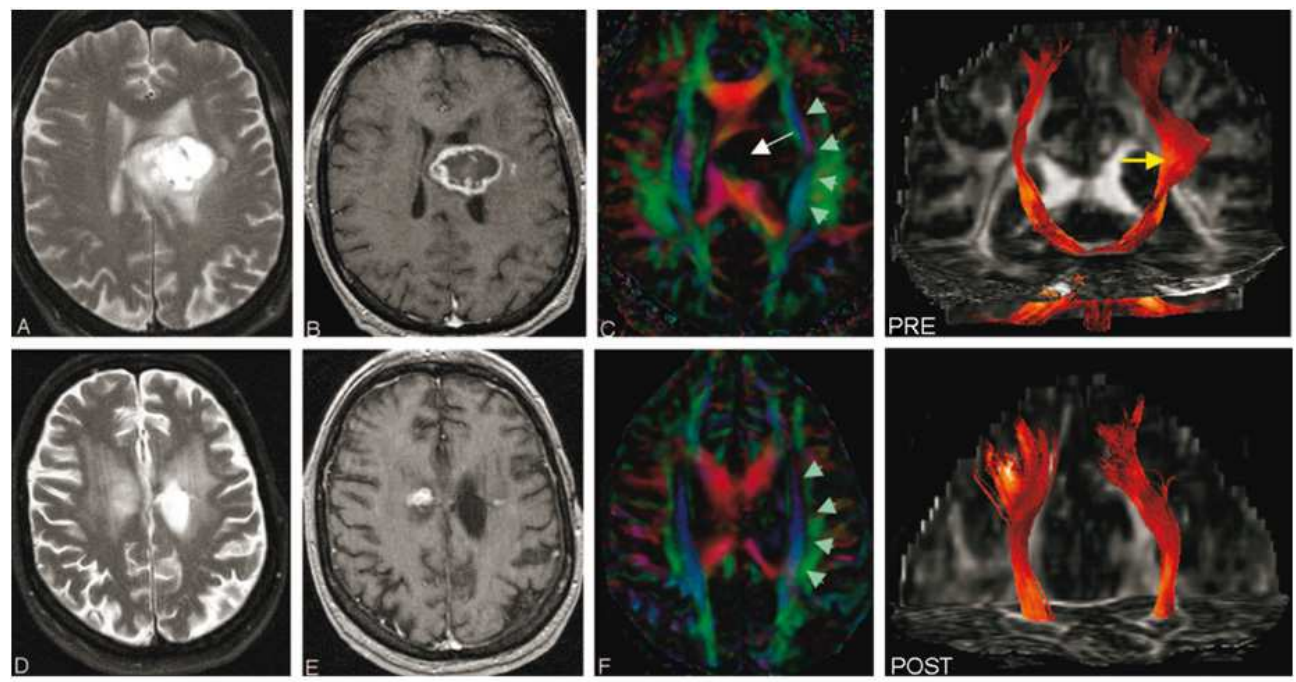

Fig. 6. MR images of a grade III glioma in the superior medial region of the left frontal lobe of a patient before (upper panels) and after (lower panels) surgery. A, D: T2W images, B, E: Gdenhanced T1W images, C, F: anisotropy weighted color maps allow differentiation of WM fiber tracts (green arrows) located in the tumor (white arrow) vicinity, PRE, POST: tractograms of the cortico-spinal tract. Yellow arrow signals deviation and deformation of the tract in the tumor proximity. (Lazar, Alexander et al. 2006). Reproduced with permission.

As shown in figure 6, DTI is probably one of the most useful clinical applications in this direction, but one of the major obstacles for accurate reconstruction of white matter trajectories is the use of an adequate and suitable tracking algorithm. Simple tracking algorithms are not appropriate in anatomically complex fascicular pathways often leading to incorrect directional information. To solve this, sophisticated algorithms have been developed to improve reconstruction of fiber tracts in these complex areas. Even more, one of the main challenges in DTI fiber tractography studies is to have the skill to reconstruct white matter tracts related to a specific network. This becomes even more complicated in the presence of a tumor where functionally relevant regions are displaced or infiltrated in the surrounding tissue. That goal has encouraged some authors to develop strategies by combining functional MRI and DTI (fMRI-guided DTI) to define related structures based on functional anatomy for subsequent fiber tracking (Kleiser, Staempfli et al.). Obviously, the ability to extract the most useful information from DTI acquisition in diagnosis, prognosis and therapy validation of gliomas is an active and challenging area of interest in clinic and research.

\subsection{Assessment of treatment response with diffusion MRI}

Since ADC measurements are able to monitor cancer progression, they may be also sensitive to assess the effectiveness to therapy. An increase in the translational motion of water molecules is expected when there are physiological responses like a loss of membrane integrity, a decrease in cellularity, changes in cellular size, extracellular volume or membrane permeability (Hamstra, Rehemtulla et al. 2007). Even more, changes in ADC 
have been reported to be dose dependent either on chemotherapy or radiotherapy in human brain tumors (Chenevert, Stegman et al. 2000). Concluding, by carrying out DWI studies and determining diffusion coefficients in glioma patients it is possible to distinguish different regions within the tumor, not accessible to conventional MRI techniques as well as to obtain an early prediction of the therapeutic efficacy.

\subsubsection{Functional Diffusion Maps}

More recently Functional Diffusion Maps (fDMs) were developed as a new application of DWI that can be used as a surrogate biomarker for brain tumor cellularity (Ellingson, Malkin et al. ; Moffat, Chenevert et al. 2005). fDMs obtained computationally allow establishing the correlation between water diffusivity and cellularity mainly to predict the effectiveness of tumor treatment on a voxel-by-voxel pixel analysis. These color based maps are obtained by comparing diffusion MR images at two time points, before and after therapy, to evaluate quantitatively the therapeutic-induced changes in ADC values of the tumor. These maps are depicted in three colors by segmenting the tumor in different regions depending on the magnitude and directions of the ADC changes, allowing the use of this computational analysis as a surrogate marker of the early tumor response to the treatment as depicted in figure 7.

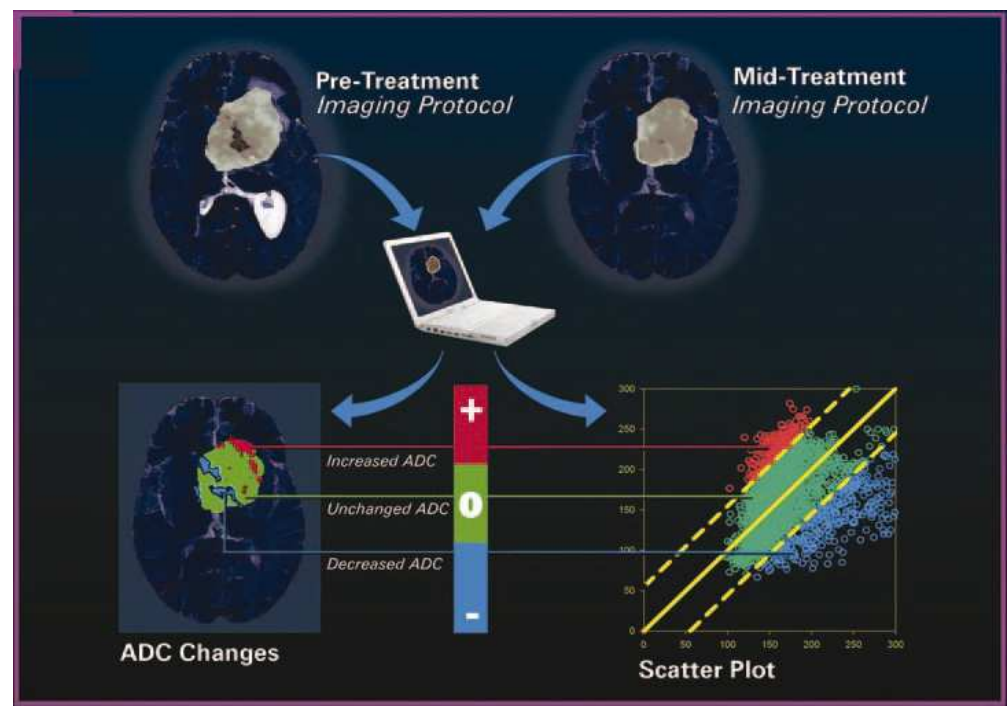

Fig. 7. fMD map involving coregistration of images before and after the treatment. A threecolor overlay of postprocessed and analyzed maps show ADC values in the tumor that are unchanged (green), significantly increased (red), or significantly decreased (blue). These regions may be associated to unresponsive (green) or responsive (red, blue) regions. Data can be also represented in a scatter plot allowing quantitative assessment of ADCs changes (Moffat, Chenevert et al. 2006). Reproduced with permission.

Some authors have concluded that fDMs as obtained from the analysis of longitudinal diffusion images appear as a potent tool to detect the therapeutic response of tumors 
through the early detection of induced changes in their morphology (Moffat, Chenevert et al. 2006; Hamstra, Galban et al. 2008). A patient with a brain tumor that shows significant changes in its ADC values after treatment is supposed to be responsive to therapy. There is the hypothesis that the water liberated by cell necrosis as induced by therapy is the major mechanism for the induced ADC increase. So, the magnitude of change in tumor water diffusion as detected by MRI is related to the quantity of cells killed by therapy and hence, to its efficacy. These changes precede the volume changes in the tumor by several weeks, providing the main justification for DWI as an early predictor of treatment response in individual patients. Even more, no changes in diffusion coefficient indicate a therapeutically unresponsive tumor suggesting the prescription of an alternative therapeutic option.

\section{Perfusion magnetic resonance imaging}

Clinical perfusion MRI measurements are currently recognized as one of the most powerful tools to assess tumor vascularisation and responses to treatment through the evaluation of hemodynamic parameters. MRI approaches to measure perfusion, can be divided in two broad classes: those monitoring tissue signal changes using an exogenous relaxation contrast agent and those that use endogenous contrast. MR perfusion weighted imaging (PWI) allows for the noninvasive estimation of tumor grade preoperatively. This can help by directing the surgeon to biopsy the most aggressive portions of the neoplasm. In addition, perfusion MR provides an exquisite delineation of tumor margins and it is useful in the follow-up of brain tumor patients after treatment by allowing differentiation between radiation effects and recurrent tumor zones. Looking into the future, perfusion changes in brain tissues hold promise as surrogate markers of response to therapy in clinical trials of new antiangiogenic drugs.

\subsection{Physiological principles}

Histopathology as gold standard of tumor grading suffers of several limitations. Mainly, since just a few samples of tissue are obtained after biopsy, the most malignant regions may be missed. Neovascularization is one of the most important criteria of malignancy for glioma grading. Malignant astrocytomas recruit existing vessels in the microenvironment and lead to form new vascular networks to supply the nutrients and oxygen required for tumor growth and proliferation. Hence, it is widely accepted nowadays the importance of neoangiogenic pathways in the treatment of brain tumors, since tumor growth of a few millimeters, involves the development of sufficiently competent networks of vascular supplies (Folkman 1971). The net result is a complex system of abnormal vessels in the peritumoral space, characterized by abnormal blood flow and increased permeability to macromolecules, both factors being potentially used as biomarkers for the evaluation of tumor growth. Concomitantly, tumor vessels are more tortuous than the healthy brain, increasing the distance that blood must travel as it moves through the tumor. Perfusion abnormalities are therefore detected not only because the increased number of vessels in gliomas but also for the aberrant reaction of those vessels to the environment. Under these circumstances, disruption of angiogenesis plays an important role in established therapies of gliomas and in the development of new ones. 


\subsection{Techniques and methods}

Currently, there are three principal methods to measure hemodynamic perfusion parameters by using MRI: T2*-weighted DSC, ASL, and T1-weighted DCE acquisitions, respectively. Any of them involves the serial acquisition of images through the brain as magnetically labelled blood passes through it. The labelling procedure can be either based on an exogenous contrast material, typically a paramagnetic complex of gadolinimum $(\mathrm{Gd})$, or on an endogenous magnetic label. Among them, $\mathrm{T} 2{ }^{*} \mathrm{~W}$ dynamic susceptibility imaging is probably the most consolidated method to carry out MR perfusion in clinical environments not only in brain oncology but also in many other pathologies, mainly neurodegenerative diseases.

\subsubsection{Dynamic Susceptibility Contrast}

Nowadays, PWI employing DSC techniques is one of the most relevant procedures of functional MRI with an increasing number of clinical applications depending on software and hardware availability. DSC enhanced images allow for the evaluation of regional cerebral hemodynamics by analyzing changes in the intensity of the MR signal during the first pass (and often second) of a narrow and concentrated bolus of an exogenous CA, through the brain microvascular bed. This technique is often called bolus tracking and performs perfusion measurements employing very rapid imaging to capture the first pass of a rapidly injected intravenous contrast material (Rosen, Belliveau et al. 1990), exploiting the $\mathrm{T}^{*}$ susceptibility effects induced by the large local concentrations of the agent rather than the T1 shortening effects routinely used in contrast enhancement by conventional MRI. For accurate assessment of the circulation it is necessary to measure signal drop during the passage of the bolus with sequences endowed with high sensitivity and temporal resolution to the magnetic susceptibility changes occurring in the tissue during the pass of the agent. One approach is to use EPI sequences enabling acquisition times of less than one second because the whole raw data set of an image is acquired after a single spin excitation. EPI is merely a read-out technique and can be combined with any technique of spin excitation (such as spin-echo or gradient echo). Due to the special readout scheme of EPI, and because major parts of k-space are affected by $\mathrm{T} 2^{*}$, each EPI sequence is inherently $\mathrm{T} 2 *$ sensitive.

In the brain, the first-pass extraction of the agent is zero when the blood-brain barrier (BBB) is intact, and the complete intravascular compartmentalization of the contrast creates strong, microscopic magnetic susceptibility gradients. These gradients cause dephasing of spins as the spins diffuse through the vessel. In general, pulse sequences without complete refocusing of static field inhomogeneities, as such gradient-echo (GE) will suffer a general signal loss due to the presence of microscopic field disturbances in the vessels. In terms of relaxivity, the shortening of $\mathrm{T} 1$ values affects only those protons near the unpaired electron clouds of the agent. This is a short-range effect. A distinct feature of the signal loss on $\mathrm{T} 2{ }^{*} \mathrm{~W}$ images is that all protons near the CA are affected because the magnetic susceptibility effect spreads far beyond the immediate vicinity of the agent. This is considered a long-range effect. However, direct damage of the BBB in a tumor may cause a disruption in it and a leakage of contrast material, a circumstance indicating that the factors responsible of the observed MR signal may be more complex.

Experimentally, the contrast medium at high concentrations (narrow bolus), induces $\mathrm{T} 2$ * shortening yielding firstly a signal loss, followed by a recovery as the agent is diluted in 
tissues (i.e. healthy brain and tumor). So, the bolus can be tracked by the acquisition of a multislice series of $\mathrm{T} 2{ }^{*}$-sensitive images from which a graph of relative signal intensity against time can be composed showing the pixel by pixel effects of the CA transit in the image. There is an approximate linear relationship between tissue contrast agent concentration and change in $\mathrm{T} 2 *$ relaxation rate, and from the signal-time course, the concentration-time course of the contrast medium can be calculated with the expression:

$$
\mathrm{Ct}(\mathrm{t})=-1 / \mathrm{TE}\left[\ln \left(\mathrm{St}-\mathrm{S}_{0}\right)\right]
$$

where $C t(t)$ is the concentration of the agent, $S t$ is the signal intensity at any time point, $S_{0}$ is the basal signal intensity before the CA injection and TE the echo time. The concentration of contrast medium is linearly proportional to changes in rate of relaxation T2* $(\mathrm{R} 2 *)$. MR data can then be converted to $\Delta \mathrm{R} 2^{*}$ versus time evolution and these values can be fitted to a gamma variate function to correct for tracer recirculation. Assuming uniform arterial concentration profiles in all arterial inputs, relative cerebral blood volume (CBV) measurements are determined simply by integrating the area under the concentration time curve (figure 8). The peak integral (that is, the peak area under the curve) is taken as the relative CBV because this represents the volume of the effect. Other parameters of interest that can be measure in the curve are the time to peak (TTP) and the mean transit time (MTT), although the true MTT requires knowledge of the input function of the bolus. Figure 8 depicts a simulation of the process.
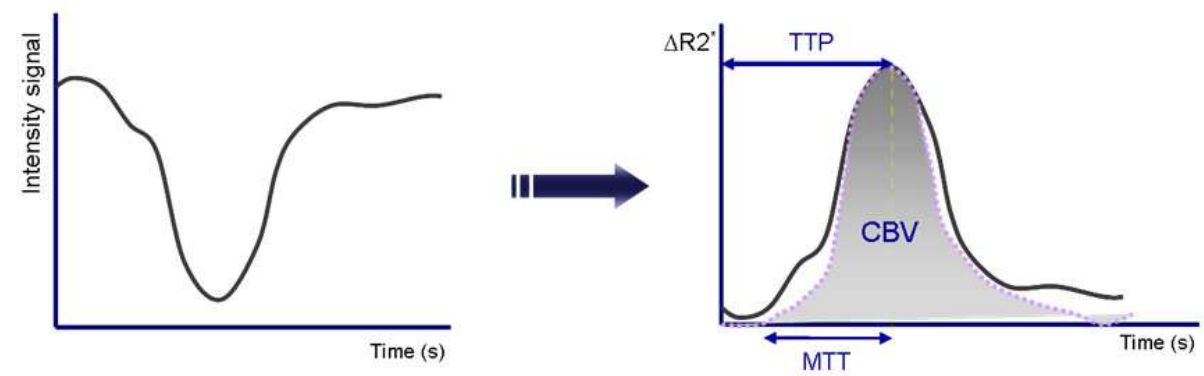

Fig. 8. Schematic behaviour of MR signal intensity as CA passes across the imagin plain in brain tissue

In this way, post-processing of DSC enhanced MR images allows mapping CBV, cerebral blood flow (CBF) and MTT by using singular value decomposition and deconvolution (Ostergaard, Weisskoff et al. 1996). Regional CBV (rCBV) is defined as the total volume of blood traversing a given brain region, measured in milliliters of blood per gram of brain tissue $(\mathrm{mL} / \mathrm{g})$. Regional CBF ( $\mathrm{rCBF}$ ) is defined as the net volume of blood traversing a given brain region per unit time, measured in milliliters of blood per gram of brain tissue per minute $(\mathrm{mL} / \mathrm{g} / \mathrm{min})$. The definition of regional MTT is more complex, but it can be described as the average amount of time it takes any water molecule or particle of CA to pass through the capillary bed, that is, to transverse between arterial inflow and venous outflow measured in seconds (s) (Covarrubias, Rosen et al. 2004). rMTT is equal to the ratio $\mathrm{rCBV} / \mathrm{rCBF}$ and although CBV may be measured with other methods, MTT measurements necessarily rely on techniques sensitive to motion. 
The higher vascularity of gliomas is usually assessed with PWI methods by measuring CBV in the tumor. A potential problem with the use of these techniques arises in regions of severe BBB disruptions as it happens in high-grade gliomas. The leakage of the contrast medium into the extravascular space during its first passage across the tumor capillary bed, increases signals above baseline due to the T1 shortening effect of the paramagnetic label used. Since the algorithm used to calculate CBV assumes a constant baseline, this signal increasing relies in an area above the concentration curve of CA versus time (left panel in figure 8) interpreted as a negative blood volume. This may yield a significant underestimation of CBV in gliomas (Aronen and Perkio 2002). Several improvements and multiple methods have been proposed to address this problem. One of the most extensively used in clinical settings is the presaturation of the extravascular extracellular space in the lesion with the preinjection of a low dose of CA $(0.05 \mathrm{mmol} / \mathrm{Kg})$ before starting perfusion MR acquisition with a normal dose of Gd (typically $0.2 \mathrm{mmol} / \mathrm{Kg}$ ) intravenously injected at a high rate. Another approach consists in using a dysprosium (Dy) based contrast medium as a paramagnetic metal with stronger T2* effects than Gd but only slight capability in T1 shortening (Vander Elst, Roch et al. 2002). A third option to reduce T1 effects goes through increasing the repetition time (TR) which reduces T1 effects but increases total acquisition time, thus reducing temporal resolution.
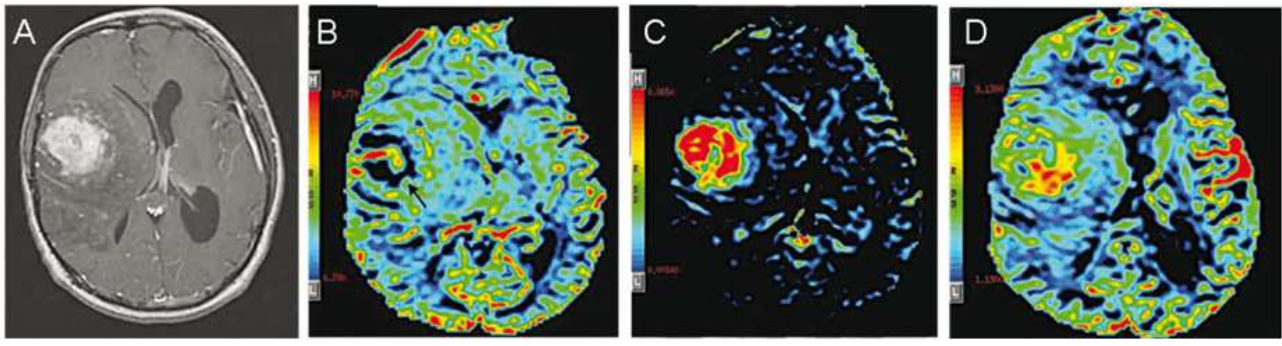

Fig. 9. Correction of CBV underestimation in a high-grade glioma. A: T1W image (Gd 0.2 $\mathrm{mmol} / \mathrm{Kg}$ ) shows an enhanced mass. B: Uncorrected CBV map shows low CBV within the lesion (black arrow). C: K2 first-pass permeability map shows increased permeability within the lesion, consistent with severe BBB breakdown. D: Corrected CBV map shows increased $\mathrm{CBV}$ within the lesion, as expected for a high-grade neoplasm (Covarrubias, Rosen et al. 2004) Reproduced with permission.

In addition, different computational approaches have been reported to avoid the CBV underestimation. In these methods, the images are mathematically corrected to account for extravasation of contrast material in regions of BBB breakdown under the premise that the observed Gd concentration can be divided in intra- and extravascular components (Aronen and Perkio 2002; Provenzale, Wang et al. 2002). With these methods, based in a twocompartmental model, permeability can be estimated in regions of BBB disruption by generation of permeability (K2) maps that can be used to correct CBV as shown in figure 9. Although DSC has the potential to allow quantitative imaging of perfusion parameters at a high signal to noise ratio in a short scan time, quantification of MTT and CBF depends critically on accurate and precise measurement of an arterial input function (AIF). The AIF relies on the measurement of the signal response in the artery or arteries that feed the tissue 
of interest. This is one factor that limits the ability to quantitate hemodynamic parameters from DSC studies. To measure AIF the imaging planes through the brain have to be chosen to contain a major intracranial artery feeding the tissue, typically one paraclinoid internal carotid artery or the middle cerebral artery (Covarrubias, Rosen et al. 2004). Then, a deconvolution algorithm is used to post-process the data and generate CBF and MTT maps. However, the absolute determination of hemodynamic parameters in not a crucial factor in grading human gliomas, the resulting ratios being equally valid for this purposes since they incorporate the measurement errors from both values.

\subsubsection{Dynamic Contrast Enhancement}

Dynamic contrast-enhanced T1W MRI (DCE-MRI) was developed in the 1990s for estimating BBB leakage (Tofts and Kermode 1991). It is entirely an approach to measure perfusion based on the T1 shortening caused by the infusion of a paramagnetic contrast media. The main objective of this technique is to measure tumor permeability by dynamic $\mathrm{T} 1 \mathrm{~W}$ imaging after the intravenous administration of a contrast medium. Typically, a single lower dose $(0.1 \mathrm{mmol} / \mathrm{Kg})$ and a lower rate than in DSC studies is injected to the patient followed by repetitive image acquisitions (every 15-30 seconds) through the tumor up to 510 minutes rather than the first pass of the bolus. The method is focussed in allowing the leakage of the CA into the extravascular space and coming into equilibrium after several passes of the bolus through the microvascular tumor bed.

In this sense, one of the main advantages of this method is that the extravasation of the contrast material through the BBB is not an artefact to be corrected. Dynamic T1W MRI acquisitions afford better visualization of tumoral heterogeneity in gliomas, improving the accuracy of the technique to detect tumor staging, tumor recurrence and follow-up response to therapies.

Appropriate information from DCE images requires measuring the real T1 values of the tissues to take them as a baseline to perform an accurate pharmacokinetic analysis (Evelhoch 1999). To get an optimal temporal resolution, three dimensional imaging schemes are typically used obtaining the arterial input function in the centre of the acquisition volumes. Hence, data acquisition parameters may influence data analysis and it is necessary to optimize them. Several post-processing methods have been developed to measure perfusion in gliomas using DCE approaches. M. Pauliah et al. described an improved procedure to perform cerebral perfusion maps using a spoiled gradient-recalled echo sequence and reporting not only morphological but also functional information in a single imaging acquisition (Pauliah, Saxena et al. 2007). Authors estimate a quantitative evaluation of both regional $\mathrm{CBV}$ and $\mathrm{CBF}$ using singular value decomposition (SVD)-based deconvolution techniques in which the concentration of contrast material passing through a given voxel can be expressed as a convolution of the AIF with the residue function tissue concentration (Ostergaard, Sorensen et al. 1996; Ostergaard, Weisskoff et al. 1996). Figure 10 shows one particular case from this study.

In summary, DCE is a powerful tool capable of providing quantitative assessment of contrast uptake and characterization of microvascular structure in human gliomas. Even more, several comparative studies have reported that DCE MRI studies of tumour enhancement in glioma patients can be used for repeated studies with reasonable reproducibility (Jackson, Jayson et al. 2003). 


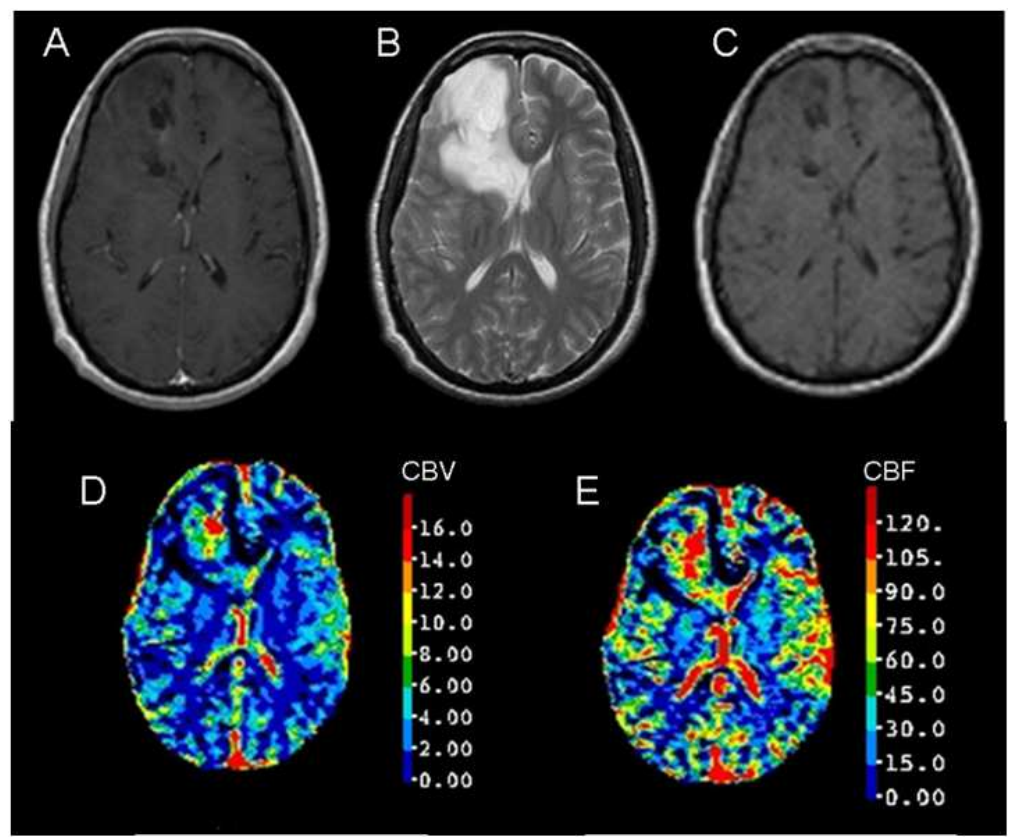

Fig. 10. DCE images of a patient with histologycally proved astrocytoma grade III. A: Post Gd-injection T1W image. B: T2W image. D: T1W image (without CA administration). C: $\mathrm{CBV}$ and $\mathrm{E}: \mathrm{CBF}$ maps generated

\subsubsection{Arterial Spin Labelling}

ASL perfusion imaging in the brain adds a new functional dimension to non invasive evaluation of the brain functionality with MRI. It is the only method that yields quantitative monitoring of cerebral perfusion over a period of several hours. Its non invasive nature allows an unlimited number of repeated measurements to be made with a high temporal resolution. ASL uses spatially selective inversion of the inflowing arterial blood magnetization as a method to label water molecules in blood. So, in a typical experiment the arterial blood is tagged and after a given delay, this tagged blood arrives at the imaging plane and an image is acquired. The MRI signal from inverted magnetization is made negative relative to uninverted one. When the labelled blood reaches the tissue, it attenuates the pre-existing signal from the image of that tissue. Therefore, a control measurement is required without tagging the arterial blood. The difference in signal intensity between both images is proportional to $\mathrm{rCBF}$, through a calibration factor that depends primarily on global properties of blood. A schematic diagram of the process is shown in figure 11. This difference signal measures how much of the original arterial magnetization created by the inversion pulse has been delivered to the voxel and survives to the time of measurement. The magnetization difference (control minus taged) carried out into the voxel can be conceptually similar to a contrast material delivered to it by CBF. Under this assumption the same kinetic modelling arguments used in bolus tracking or DSC studies can be applied to ASL (Buxton, Frank et al. 1998). Nevertheless, several premises remain to be taken into account for an accurate 
quantification of CBF by using ASL. The method necessarily has to: i) perform an accurate control measurement; ii ) create a well-defined tagged bolus of arterial blood, waiting sufficiently long for the bolus to be delivered to the imaging plane; and iii) account for relaxation of the magnetization label (Buxton 2005).
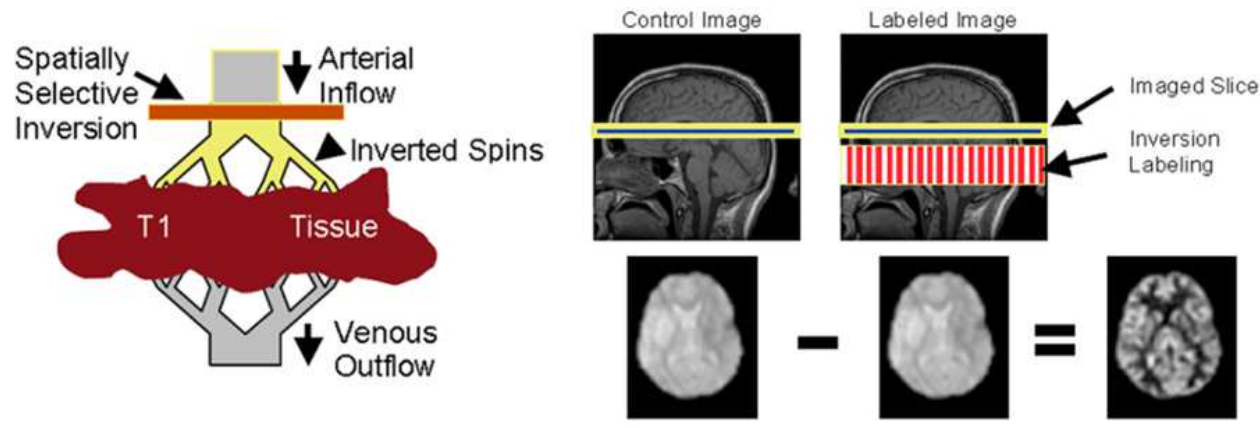

Fig. 11. Fundamentals of the ASL method: Arterial blood is tagged by inverting the magnetization and, after a delay, it arrives to imaging plane decreasing its intensity in manner proportional to blood flow (taken from "ASL Perfusion Imaging: Concepts and Applications", David C. Alsop., ISMRM-2006). Reproduced with permission.

ASL is an attractive and ingenious method for clinical applications because it does not use an intravenous contrast agent, but merely a manipulation of the proton spin of water molecules within the vasculature, that has the potential to provide a robust estimation of CBF. Hence, the agent used in ASL for assessing perfusion is labelled water and not a paramagnetic or superparamagnetic contrast medium, potentially affecting the physical, chemical or physiological properties of the blood. Another advantage of this approach is that it is independent of tumor permeability, without the need to perform additional corrections in the post-processing of the data. The perfusion map obtained by difference of images is available just after acquisition with minimal post-processing, representing a clear benefit in routine clinical settings. On the other hand, ASL leads reliable absolute quantification of $\mathrm{CBF}$ that is not affected by BBB damages.

The ASL methods apply a powerful magnetic gradient to invert the inflowing blood magnetization tagging it as it flows upstream. ASL techniques can be divided in two main classes, depending on how the tag is applied: continuous (CASL) or pulsed (PASL). In CASL the inflowing arterial blood is continuously labelled bellow the slab to be imaged until the tissue magnetization gets a steady state (Petersen, Zimine et al. 2006). In PASL the spin inversion is achieved using short inversion pulses in a thick slab located next to the slice of interest. This method is easier to implement and has less practical problems as compared with CASL (Petersen, Zimine et al. 2006). Interestingly, PASL does not suffer as many magnetization transfer effects as CASL methods, and the selective inversion applied is closer to the image slices yielding smaller signal attenuation because of the reduced transit delay and a better inversion efficiency. Even more, short inversion pulses minimize problems of energy deposition in humans at high fields. 
ASL has been also used as a functional method to detect CBF changes in local CBF during task activation as a surrogate marker or neuronal activity. This can be very helpful in the assessment of gliomas. To investigate if ASL methodologies contribute measurements that can be used as reliable markers of tumor grade, several studies have compared the perfusion results obtained by MRI with histological data in glioma patients (Warmuth, Gunther et al. 2003; Wolf, Wang et al. 2005). As perfusion MR leads to images of the entire neoplasm, these can help in characterizing the malignancy in gliomas overcoming some of the limitations of histopathology sampling. In this line, ASL perfusion images allow delineating tumor heterogeneity and this can be used to guide biopsy towards the most malignant region of the tumor. Some examples of perfusion maps obtained from ASL acquisitions in gliomas are shown in Figure 12.
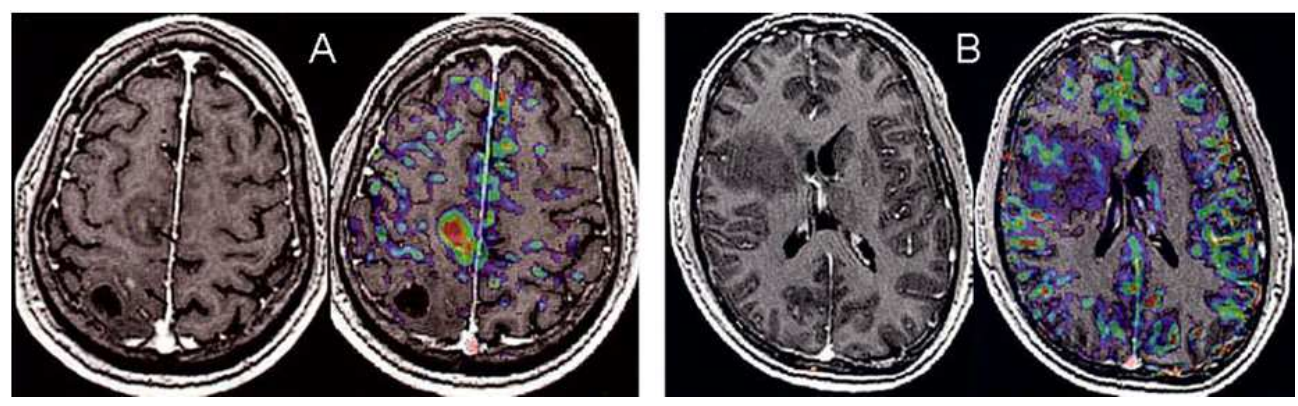

Fig. 12. Differential diagnosis of gliomas using ASL perfusion. A: Giant cells glioblastoma. B: Anaplastic astrocytomas. In both, left: post-contrast T1W; right: perfusion map from PASL (Tourdias, Rodrigo et al. 2008). Reproduced with permission.

A close relationship has been established between ASL and bolus tracking methods in determining $\mathrm{CBF}$ in brain tumors, been both approaches able to differentiate between high-grade and low-grade gliomas. Probably, the main limitation of using ASL sequences is that they only allow for the calculation of $\mathrm{CBF}$, whereas MR perfusion measurements based on the administration of a contrast material can provide additionally, CBV and MTT values by using DSC methods (T2*W based images), and permeability values by DCE sequences (T1W based images). This additional information may contribute appreciably to the characterization and grading of gliomas. On the other hand, comparative studies in glioma patients have shown that the ASL technique is more suitable than exogenous contrast-based perfusion analysis for assessing hypervascularized lesions, whereas it has a bigger error rate in the assessment of hypovascularized ones (Warmuth, Gunther et al. 2003).

\subsection{Tumor grading by perfusion MRI}

Current methods to grade gliomas have inherent limitations, the infiltrative proliferation as an intrinsic feature of the tumor becoming a significant problem. Conventional MR images with CA provide important information regarding Gd-based enhancement that is useful in characterizing tumor grade. The most malignant enhancing brain tumors include 
high-grade gliomas, metastases, and lymphomas. These methods assign the most aggressive and malignant grade to the most enhanced tumors, but regarding the gliomas, this is not always reliable. A high-grade glioma may be often mistaken for a low-grade one when images show minimal edema, no gadolinium enhancement, no necrosis and no mass effect. Equally, low-grade gliomas can depict sometimes peritumoral edema, contrast enhancement, necrosis and mass effect leading to a high-grade false diagnosis (Law, Yang et al. 2003). In histopathology grading of gliomas, tumor cellularity and vascularity are the major factors to be taken into account. This information is accessible by the advanced functional MRI methodologies detailed in this chapter and can help in brain tumor grading. As regarding perfusion-based maps it is widely accepted that high regional $\mathrm{CBV}$ is more likely in high-grade gliomas. This parameter can reflect tumor vascularity and be used as an indicator of histological grade. In fact, many groups have shown the ability of DSC MRI for estimating the histopathological glioma grade. Several studies reported rCBV values measured in a large number of brain tumor patients suggesting that this parameter can improve distinction between high-grade and lowgrade gliomas (Aronen, Gazit et al. 1994; Calli, Kitis et al. 2006) with a high true-positive rate and a very low false-negative rate. On the other hand, gliomas have a great histopathological variability and in this sense, perfusion maps allow also for the localization of foci with very high rCBV values within the tumor. PWI can thus be very helpful not only for a valuable preoperative grading, but also for guiding the surgeon to the most appropriate site to obtain a representative biopsy of the most malignant portion.

Although CBV is the most commonly used hemodynamic parameter in glioma grading, the largest limitation, entailing special clinical relevance, is the lack of discrimination between anaplastic gliomas and glioblastoma multiforme, the two most aggressive tumors of the high-grade glioma group. Perfusion MRI studies report no significant difference in rCBV for both of them (Covarrubias, Rosen et al. 2004).

\subsection{Assessment of permeability with perfusion MRI}

A link between permeability, angiogenesis and malignancy in gliomas is normally assumed. Several perfusion MRI studies suggest a correlation between permeability and malignancy, but they do not establish a link between angiogenesis and permeability or angiogenesis and malignancy. What is widely accepted is that high permeability to macromolecules, due to the presence of endothelial gaps in tumor vasculature, is an important mediator of tumor growth and a pathognomonic feature for malignancy. Permeability studies are typically based in the determination of the transfer constant of contrast between the plasma and the extravascular extracellular space (Ktrans). Measurements of $\mathrm{K}^{\text {trans }}$ are increasingly popular as a method for the quantification of contrast enhancement since they are designed to describe the distribution of contrast agent, free from scanning and machine dependant variables, which allow comparison of results from different studies and imaging centers. Nowadays, perfusion MRI parameters such as $\mathrm{K}^{\text {trans }}$ and $\mathrm{CBV}$ can be directly correlated with histopathological changes in the brain.

T1W dynamic contrast MRI is probably the preferred method to analyze vascular permeability in gliomas and for mapping tumor angiogenesis. There are important studies showing a strong correlation between tumor grade and microvascular permeability by using DCE imaging methods (Roberts, Roberts et al. 2002). The net result is that blood vessel 
permeability in gliomas can be used as a non invasive surrogate marker able to provide information related to rate of angiogenesis in the tumor. High-grade gliomas depict high vascular permeability, a consequence of the massive BBB disruption leading to extravasation of contrast material into the extravascular space that becomes detectable in MR images.

Although studies in humans have been typically carried out with DCE methods, it is also possible to perform and compare permeability measurements in grading gliomas employing T2* DSC techniques, within certain limitations (Provenzale, Wang et al. 2002). In this case, the $\mathrm{K}^{\text {trans }}$ maps can be generated by employing a calculation that allows the separation of $\mathrm{T} 1$ effects from the analysis. The advantage of using this method is that it is possible to obtain CBV and permeability maps from the same data set, providing simultaneously, two types of valuable information.

\subsection{Assessment of treatment response with perfusion MRI}

At present, the predominant method to assess tumor response to treatment is to measure the size of the lesion by using conventional, paramagnetically enhanced MRI. However, tumor size is not always specific of tumor behaviour and neoplastic features associated with aggressiveness of the tumor may vary without changes in the tumor size. On the other hand, since angiogenesis plays a critical role not only in the development of brain tumors but also in the malignant transformation, many of the novel therapies currently investigated are focused on antiangiogenic strategies. Monitoring the success of such therapeutic methods relies on the ability to quantify tumor vascularization through the measurements of hemodynamic parameters. It is widely accepted now that the use of quantitative measurements of tissue enhancement characteristics provides a more valuable indicator to monitor the therapeutic response of tumours to novel antiangiogenic drugs in the treatment of gliomas. For example, rCBV is supposed to reflect microvessel density, an early response in this parameter to antiangiogenic therapies should show a decrease, thus providing the potential to predict growth or regression.

Figure 13 shows a clear example of this behaviour. While T1W images show tumor growth even 6 weeks after initiating the treatment, rCBV maps from PWI depict a much earlier drop in tumor perfusion. This demonstrates that tumor size and perfusion may lead opposite conclusions because they represent indeed, different tumor features.

Because the increase in endothelial permeability is a primary effect of vascular endothelial growth factor (VEGF) and other angiogenic cytokines, changes in the capillary leakage of contrast media may offer a predictable response to anti-angiogenic therapies. Quantification of the enhancement effect is therefore a good candidate as a potential biomarker of drug activity. Changes in permeability occur rapidly in response to VEGF inhibitions, so assessment of these changes either by DSC or DCE can be used as a marker of antiangiogenic therapy responses. Although quantification of permeability would be an optimal strategy, this is in fact, extremely difficult to achieve. Along these lines, several pharmacokinetic models to analyze dynamic enhancement data have been developed to estimate $\mathrm{K}^{\text {trans }}$ and used it as an therapeutic indicator (Jackson, Jayson et al. 2003). Now, it is accepted and recommended the use of perfusion MRI to monitor tumor angiogenesis and evaluate the response to antiangiogenic therapies, a subject that has gained considerable clinical and research efforts, even leading to published recommendations concerning this important capacity of MRI (Brasch, Li et al. 2000). 


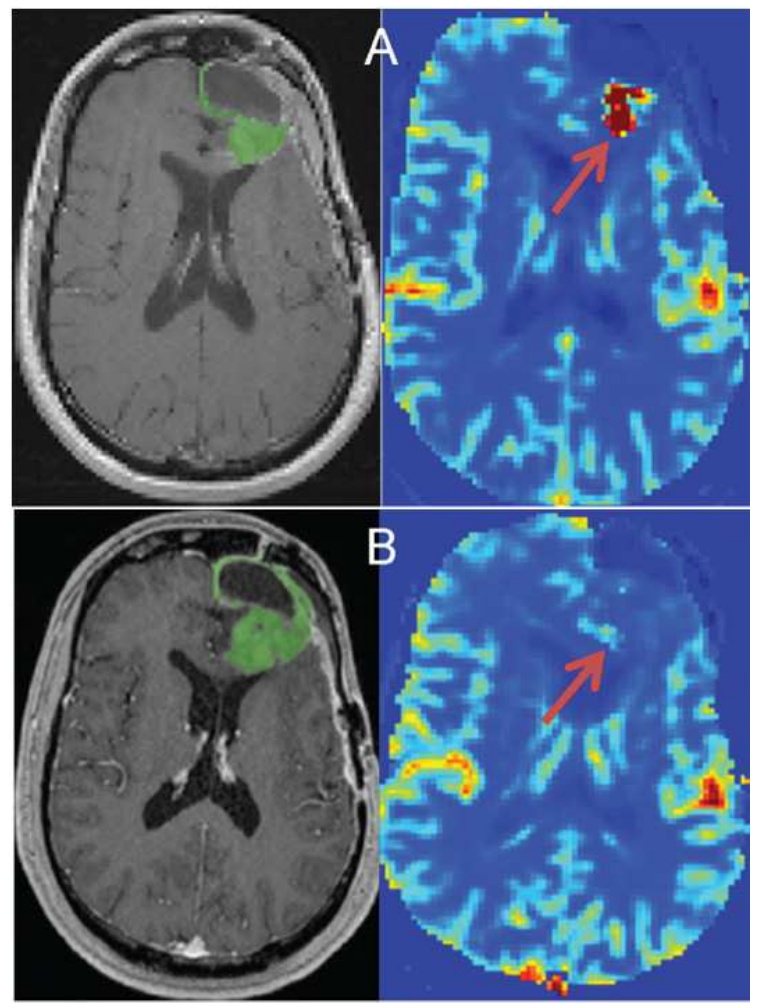

Fig. 13. Change in perfusion in a pathologically confirmed GMB case before (A) and after (B) the initiation of anti-angiogenic chemotherapy. Left images in both panels show contrast enhanced T1W images used to measure tumor volume (green). Right images depict CA leakage corrected rCBV maps showing tumor perfusion (red arrow) (Sawlani, Raizer et al.) Reproduced with permission.

\section{Concludig remarks and future perspectives}

In summary, we presented an overview of the fundamentals and clinical applications of advanced Magnetic Resonance Imaging protocols providing in situ information on water diffusion and blood perfusion in gliomas. These methodologies have shown considerable ability to provide functional information on the malignancy of these lesions as well as in its response to therapy. Together, they allow for a more adequate patient management based fully in non invasive non destructive examinations. These MRI approaches have been, however, applied independently in most cases. The future use of combinations of these two strategies may offer the possibility to compensate the limitations of one technology with the strengths of the other, still maintaining their non invasive character. In this sense, combined diffusion-perfusion methodologies may provide in the future significant advances, becoming even more effective in clinic for the diagnosis, prognosis and therapy assessment of gliomas. 


\section{Acknowledgements}

This work was supported in part by grants CTQ2010-20960-C02-02 to P.L.L. and grant SAF2008-01327 to S.C. A.M.M. held an Erasmus Fellowship from Coimbra University and E.C.C. a predoctoral CSIC contract. The valuable contribution of Mr. Javier Perez drafting the illustrations is gratefully acknowledged.

\section{References}

Aronen, H. J., I. E. Gazit, et al. (1994). "Cerebral blood volume maps of gliomas: comparison with tumor grade and histologic findings." Radiology 191(1): 41-51.

Aronen, H. J. and J. Perkio (2002). "Dynamic susceptibility contrast MRI of gliomas." Neuroimaging Clin N Am 12(4): 501-23.

Basser, P. J. and D. K. Jones (2002). "Diffusion-tensor MRI: theory, experimental design and data analysis - a technical review." NMR Biomed 15(7-8): 456-67.

Brasch, R. C., K. C. Li, et al. (2000). "In vivo monitoring of tumor angiogenesis with MR imaging." Acad Radiol 7(10): 812-23.

Brunberg, J. A., T. L. Chenevert, et al. (1995). "In vivo MR determination of water diffusion coefficients and diffusion anisotropy: correlation with structural alteration in gliomas of the cerebral hemispheres." AJNR Am J Neuroradiol 16(2): 361-71.

Bulakbasi, N., M. Kocaoglu, et al. (2003). "Combination of single-voxel proton MR spectroscopy and apparent diffusion coefficient calculation in the evaluation of common brain tumors." AJNR Am J Neuroradiol 24(2): 225-33.

Buxton, R. B. (2005). "Quantifying CBF with arterial spin labeling." J Magn Reson Imaging 22(6): 723-6.

Buxton, R. B., L. R. Frank, et al. (1998). "A general kinetic model for quantitative perfusion imaging with arterial spin labeling." Magn Reson Med 40(3): 383-96.

Calli, C., O. Kitis, et al. (2006). "Perfusion and diffusion MR imaging in enhancing malignant cerebral tumors." Eur J Radiol 58(3): 394-403.

Covarrubias, D. J., B. R. Rosen, et al. (2004). "Dynamic magnetic resonance perfusion imaging of brain tumors." Oncologist 9(5): 528-37.

Chenevert, T. L., L. D. Stegman, et al. (2000). "Diffusion magnetic resonance imaging: an early surrogate marker of therapeutic efficacy in brain tumors." J Natl Cancer Inst 92(24): 2029-36.

Chien, D., R. B. Buxton, et al. (1990). "MR diffusion imaging of the human brain." J Comput Assist Tomogr 14(4): 514-20.

Detre, J. A., J. Wang, et al. (2009). "Arterial spin-labeled perfusion MRI in basic and clinical neuroscience." Curr Opin Neurol 22(4): 348-55.

Ellingson, B. M., M. G. Malkin, et al. "Validation of functional diffusion maps (fDMs) as a biomarker for human glioma cellularity." J Magn Reson Imaging 31(3): 538-48.

Evelhoch, J. L. (1999). "Key factors in the acquisition of contrast kinetic data for oncology." J Magn Reson Imaging 10(3): 254-9.

Folkman, J. (1971). "Tumor angiogenesis: therapeutic implications." N Engl J Med 285(21): 1182-6.

Furnari, F. B., T. Fenton, et al. (2007). "Malignant astrocytic glioma: genetics, biology, and paths to treatment." Genes Dev 21(21): 2683-710. 
Gauvain, K. M., R. C. McKinstry, et al. (2001). "Evaluating pediatric brain tumor cellularity with diffusion-tensor imaging." AJR Am J Roentgenol 177(2): 449-54.

Gideon, P., C. Thomsen, et al. (1994). "Increased self-diffusion of brain water in hydrocephalus measured by MR imaging." Acta Radiol 35(6): 514-9.

Hajnal, J. V., M. Doran, et al. (1991). "MR imaging of anisotropically restricted diffusion of water in the nervous system: technical, anatomic, and pathologic considerations." J Comput Assist Tomogr 15(1): 1-18.

Hamstra, D. A., C. J. Galban, et al. (2008). "Functional diffusion map as an early imaging biomarker for high-grade glioma: correlation with conventional radiologic response and overall survival." J Clin Oncol 26(20): 3387-94.

Hamstra, D. A., A. Rehemtulla, et al. (2007). "Diffusion magnetic resonance imaging: a biomarker for treatment response in oncology." J Clin Oncol 25(26): 4104-9.

Jackson, A., G. C. Jayson, et al. (2003). "Reproducibility of quantitative dynamic contrastenhanced MRI in newly presenting glioma." Br J Radiol 76(903): 153-62.

Jakab, A., P. Molnar, et al. "Glioma grade assessment by using histogram analysis of diffusion tensor imaging-derived maps." Neuroradiology.

Jenkinson, M. D., D. G. Du Plessis, et al. (2007). "Advanced MRI in the management of adult gliomas." Br J Neurosurg 21(6): 550-61.

Kinoshita, M., K. Yamada, et al. (2005). "Fiber-tracking does not accurately estimate size of fiber bundle in pathological condition: initial neurosurgical experience using neuronavigation and subcortical white matter stimulation." Neuroimage 25(2): 424-9.

Kleiser, R., P. Staempfli, et al. "Impact of fMRI-guided advanced DTI fiber tracking techniques on their clinical applications in patients with brain tumors." Neuroradiology 52(1): 37-46.

Kono, K., Y. Inoue, et al. (2001). "The role of diffusion-weighted imaging in patients with brain tumors." AJNR Am J Neuroradiol 22(6): 1081-8.

Krabbe, K., P. Gideon, et al. (1997). "MR diffusion imaging of human intracranial tumours." Neuroradiology 39(7): 483-9.

Larsson, H. B., F. Courivaud, et al. (2009). "Measurement of brain perfusion, blood volume, and blood-brain barrier permeability, using dynamic contrast-enhanced $\mathrm{T}(1)$ weighted MRI at 3 tesla." Magn Reson Med 62(5): 1270-81.

Larsson, H. B., C. Thomsen, et al. (1992). "In vivo magnetic resonance diffusion measurement in the brain of patients with multiple sclerosis." Magn Reson Imaging 10(1): 7-12.

Law, M., S. Yang, et al. (2003). "Glioma grading: sensitivity, specificity, and predictive values of perfusion MR imaging and proton MR spectroscopic imaging compared with conventional MR imaging." AJNR Am J Neuroradiol 24(10): 1989-98.

Lazar, M., A. L. Alexander, et al. (2006). "White matter reorganization after surgical resection of brain tumors and vascular malformations." AJNR Am J Neuroradiol 27(6): 1258-71.

Le Bihan, D., E. Breton, et al. (1986). "MR imaging of intravoxel incoherent motions: application to diffusion and perfusion in neurologic disorders." Radiology 161(2): 401-7.

Lu, S., D. Ahn, et al. (2003). "Peritumoral diffusion tensor imaging of high-grade gliomas and metastatic brain tumors." AJNR Am J Neuroradiol 24(5): 937-41. 
Lyng, H., O. Haraldseth, et al. (2000). "Measurement of cell density and necrotic fraction in human melanoma xenografts by diffusion weighted magnetic resonance imaging." Magn Reson Med 43(6): 828-36.

Moffat, B. A., T. L. Chenevert, et al. (2005). "Functional diffusion map: a noninvasive MRI biomarker for early stratification of clinical brain tumor response." Proc Natl Acad Sci U S A 102(15): 5524-9.

Moffat, B. A., T. L. Chenevert, et al. (2006). "The functional diffusion map: an imaging biomarker for the early prediction of cancer treatment outcome." Neoplasia 8(4): 259-67.

Mori, S. and P. B. Barker (1999). "Diffusion magnetic resonance imaging: its principle and applications." Anat Rec 257(3): 102-9.

Mori, S. and P. C. van Zijl (2002). "Fiber tracking: principles and strategies - a technical review." NMR Biomed 15(7-8): 468-80.

Nucifora, P. G., R. Verma, et al. (2007). "Diffusion-tensor MR imaging and tractography: exploring brain microstructure and connectivity." Radiology 245(2): 367-84.

Ostergaard, L. (2005). "Principles of cerebral perfusion imaging by bolus tracking." J Magn Reson Imaging 22(6): 710-7.

Ostergaard, L., A. G. Sorensen, et al. (1996). "High resolution measurement of cerebral blood flow using intravascular tracer bolus passages. Part II: Experimental comparison and preliminary results." Magn Reson Med 36(5): 726-36.

Ostergaard, L., R. M. Weisskoff, et al. (1996). "High resolution measurement of cerebral blood flow using intravascular tracer bolus passages. Part I: Mathematical approach and statistical analysis." Magn Reson Med 36(5): 715-25.

Pacheco-Torres, J., D. Calle, et al. "Environmentally sensitive paramagnetic and diamagnetic contrast agents for nuclear magnetic resonance imaging and spectroscopy." Curr Top Med Chem 11(1): 115-30.

Pauliah, M., V. Saxena, et al. (2007). "Improved T(1)-weighted dynamic contrast-enhanced MRI to probe microvascularity and heterogeneity of human glioma." Magn Reson Imaging 25(9): 1292-9.

Petersen, E. T., I. Zimine, et al. (2006). "Non-invasive measurement of perfusion: a critical review of arterial spin labelling techniques." Br J Radiol 79(944): 688-701.

Price, S. J., N. G. Burnet, et al. (2003). "Diffusion tensor imaging of brain tumours at 3T: a potential tool for assessing white matter tract invasion?" Clin Radiol 58(6): 455-62.

Provenzale, J. M., S. Mukundan, et al. (2006). "Diffusion-weighted and perfusion MR imaging for brain tumor characterization and assessment of treatment response." Radiology 239(3): 632-49.

Provenzale, J. M., G. R. Wang, et al. (2002). "Comparison of permeability in high-grade and low-grade brain tumors using dynamic susceptibility contrast MR imaging." AJR Am J Roentgenol 178(3): 711-6.

Reardon, D. A., J. N. Rich, et al. (2006). "Recent advances in the treatment of malignant astrocytoma." J Clin Oncol 24(8): 1253-65.

Roberts, H. C., T. P. Roberts, et al. (2002). "Quantitative estimation of microvascular permeability in human brain tumors: correlation of dynamic Gd-DTPA-enhanced MR imaging with histopathologic grading." Acad Radiol 9 Suppl 1: S151-5.

Rosen, B. R., J. W. Belliveau, et al. (1990). "Perfusion imaging with NMR contrast agents." Magn Reson Med 14(2): 249-65. 
Sawlani, R. N., J. Raizer, et al. "Glioblastoma: a method for predicting response to antiangiogenic chemotherapy by using MR perfusion imaging--pilot study." Radiology 255(2): 622-8.

Schaefer, P. W., P. E. Grant, et al. (2000). "Diffusion-weighted MR imaging of the brain." Radiology 217(2): 331-45.

Schluter, M., B. Stieltjes, et al. (2005). "Detection of tumour infiltration in axonal fibre bundles using diffusion tensor imaging." Int J Med Robot 1(3): 80-6.

Stejskal, E. O. and J. E. Tanner (1965). "Spin Diffusion Measurements: Spin Echoes in the Presence of a Time-Dependent Field Gradient." The Journal of Chemical Physics 42(1): 288-292.

Sugahara, T., Y. Korogi, et al. (1999). "Usefulness of diffusion-weighted MRI with echoplanar technique in the evaluation of cellularity in gliomas." J Magn Reson Imaging 9(1): 53-60.

Thomsen, C., O. Henriksen, et al. (1987). "In vivo measurement of water self diffusion in the human brain by magnetic resonance imaging." Acta Radiol 28(3): 353-61.

Tien, R. D., G. J. Felsberg, et al. (1994). "MR imaging of high-grade cerebral gliomas: value of diffusion-weighted echoplanar pulse sequences." AJR Am J Roentgenol 162(3): 6717.

Tofts, P. S. and A. G. Kermode (1991). "Measurement of the blood-brain barrier permeability and leakage space using dynamic MR imaging. 1. Fundamental concepts." Magn Reson Med 17(2): 357-67.

Tourdias, T., S. Rodrigo, et al. (2008). "Pulsed arterial spin labeling applications in brain tumors: practical review." J Neuroradiol 35(2): 79-89.

Tropine, A., G. Vucurevic, et al. (2004). "Contribution of diffusion tensor imaging to delineation of gliomas and glioblastomas." J Magn Reson Imaging 20(6): 905-12.

Vander Elst, L., A. Roch, et al. (2002). "Dy-DTPA derivatives as relaxation agents for very high field MRI: the beneficial effect of slow water exchange on the transverse relaxivities." Magn Reson Med 47(6): 1121-30.

Warach, S., D. Chien, et al. (1992). "Fast magnetic resonance diffusion-weighted imaging of acute human stroke." Neurology 42(9): 1717-23.

Warmuth, C., M. Gunther, et al. (2003). "Quantification of blood flow in brain tumors: comparison of arterial spin labeling and dynamic susceptibility-weighted contrastenhanced MR imaging." Radiology 228(2): 523-32.

Witwer, B. P., R. Moftakhar, et al. (2002). "Diffusion-tensor imaging of white matter tracts in patients with cerebral neoplasm." J Neurosurg 97(3): 568-75.

Wolf, R. L., J. Wang, et al. (2005). "Grading of CNS neoplasms using continuous arterial spin labeled perfusion MR imaging at 3 Tesla." J Magn Reson Imaging 22(4): 475-82. 


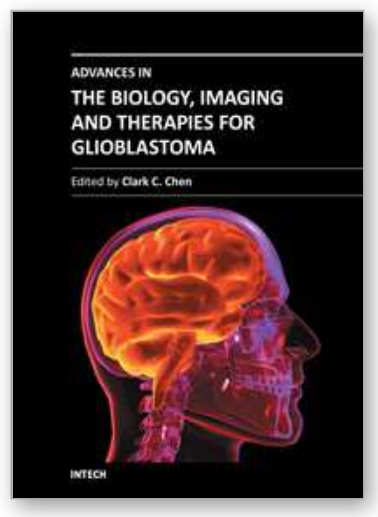

\author{
Advances in the Biology, Imaging and Therapies for Glioblastoma \\ Edited by Prof. Clark Chen
}

ISBN 978-953-307-284-5

Hard cover, 424 pages

Publisher InTech

Published online 09, November, 2011

Published in print edition November, 2011

This book is intended for physicians and scientists with interest in glioblastoma biology, imaging and therapy. Select topics in DNA repair are presented here to demonstrate novel paradigms as they relate to therapeutic strategies. The book should serve as a supplementary text in courses and seminars as well as a general reference.

\title{
How to reference
}

In order to correctly reference this scholarly work, feel free to copy and paste the following:

Pilar López-Larrubia, Eva Cañadillas-Cárdenas, Ana M. Metelo, Nuria Arias, Miguel Martinez-Maestro, Aire Salguero and Sebastián Cerdán (2011). Magnetic Resonance Imaging of Gliomas, Advances in the Biology, Imaging and Therapies for Glioblastoma, Prof. Clark Chen (Ed.), ISBN: 978-953-307-284-5, InTech, Available from: http://www.intechopen.com/books/advances-in-the-biology-imaging-and-therapies-forglioblastoma/magnetic-resonance-imaging-of-gliomas

\section{INTECH}

open science | open minds

\section{InTech Europe}

University Campus STeP Ri

Slavka Krautzeka 83/A

51000 Rijeka, Croatia

Phone: +385 (51) 770447

Fax: +385 (51) 686166

www.intechopen.com

\section{InTech China}

Unit 405, Office Block, Hotel Equatorial Shanghai

No.65, Yan An Road (West), Shanghai, 200040, China

中国上海市延安西路65号上海国际贵都大饭店办公楼 405 单元

Phone: +86-21-62489820

Fax: $+86-21-62489821$ 
(C) 2011 The Author(s). Licensee IntechOpen. This is an open access article distributed under the terms of the Creative Commons Attribution 3.0 License, which permits unrestricted use, distribution, and reproduction in any medium, provided the original work is properly cited. 